\title{
Microstructural evolution and formation of nanocrystalline intermetallic compound during surface mechanical attrition treatment of cobalt
}

\author{
X.L. Wu ${ }^{\mathrm{a}, *}$, N.R. Tao ${ }^{\mathrm{b}}$, Q.M. Wei ${ }^{\mathrm{c}}$, P. Jiang ${ }^{\mathrm{a}}, \mathrm{J} . \mathrm{Lu}{ }^{\mathrm{d}}$, K. Lu ${ }^{\mathrm{b}}$ \\ ${ }^{a}$ State Key Laboratory of Nonlinear Mechanics, Institute of Mechanics, Chinese Academy of Sciences, Beijing 100080, China \\ b Shenyang National Laboratory for Materials Sciences, Institute of Metal Research, Chinese Academy of Sciences, Shenyang 110016, China \\ ${ }^{\mathrm{c}}$ Department of Mechanical Engineering and Engineering Science, University of North Carolina at Charlotte, Charlotte, NC 28223, USA \\ d Department of Mechanical Engineering, The Hong Kong Polytechnic University, Hung Hom, Kowloon, Hong Kong
}

Received 17 April 2007; received in revised form 30 May 2007; accepted 21 June 2007

Available online 13 August 2007

\begin{abstract}
Nanocrystalline intermetallic $\mathrm{Co}_{3} \mathrm{Fe}_{7}$ was produced on the surface of cobalt via surface mechanical attrition (SMA). Deformationinduced diffusion entailed the formation of a series of solid solutions. Phase transitions occurred depending on the atomic fraction of $\mathrm{Fe}$ in the surface solid solutions: from hexagonal close-packed $(<4 \% \mathrm{Fe})$ to face-centered cubic (fcc) $(4-11 \% \mathrm{Fe})$, and from fcc to body-centered cubic $(>11 \% \mathrm{Fe})$. Nanoscale compositional probing suggested significantly higher Fe contents at grain boundaries and triple junctions than grain interiors. Short-circuit diffusion along grain boundaries and triple junctions dominate in the nanocrystalline intermetallic compound. Stacking faults contribute significantly to diffusion. Diffusion enhancement due to high-rate deformation in SMA was analyzed by regarding dislocations as solute-pumping channels, and the creation of excess vacancies. Non-equilibrium, atomic level alloying can then be ascribed to deformation-induced intermixing of constituent species. The formation mechanism of nanocrystalline intermetallic grains on the SMA surface can be thought of as a consequence of numerous nucleation events and limited growth. (C) 2007 Acta Materialia Inc. Published by Elsevier Ltd. All rights reserved.
\end{abstract}

Keywords: Nanocrystalline materials; Diffusion; Intermetallics; Surface mechanical attrition

\section{Introduction}

Nanocrystalline (nc) metals and alloys produced by methods based on severe plastic deformation (SPD) have been studied extensively over the past decade. Various SPD variants have been developed, such as equal-channel angular pressing (ECAP) [1,2], high-pressure torsion (HPT) [1], accumulated roll bonding (ARB) [3], slide loading [4] and surface mechanical attrition treatment (SMAT) $[5,6]$.

It has been recognized that nc materials exhibit superior diffusion properties $[7,8]$. From experimental results and

\footnotetext{
${ }^{*}$ Corresponding author. Tel.: +8610 62618150; fax: +86 1062561284 .

E-mail address: xlwu@imech.ac.cn (X.L. Wu).
}

theoretical models it follows that the highly enhanced diffusion kinetics in nc materials is related to the much increased portions of grain boundaries (GBs) and triple junctions (TJs) [9-17]. The GBs in nc materials by SPD are in high-energy, non-equilibrium states, making them the rapid diffusion paths $[7,10]$. Diffusion coefficients associated with such GBs could be several orders of magnitude larger than those in the nc materials fabricated by more equilibrium methods (such as hot-consolidation of nc powders), as well as in conventional polycrystallites [7]. On the other hand, TJs are believed to have a more open structure than GBs, and consequently they should have higher diffusivity [17-19]. Diffusion along TJs should dominate in nc materials with grain sizes less than $\sim 10 \mathrm{~nm}$, where the volume fraction of TJs is significantly increased [17-20]. 
It is therefore reasonable to believe that the various types of high-density defects created during SPD significantly contribute to the much enhanced diffusion kinetics in SPD nanomaterials. For example, dislocations can provide pipe diffusion mechanisms through dislocation cores serving as solute pumps $[21,22]$. The global diffusion kinetics will then be an increasing function of the dislocation density. According to the Taylor law, which links the dislocation density in a metal to its flow stress, therefore, diffusion kinetics can be accelerated by increased flow stresses. Meanwhile, plastic deformation may induce vacancies and increase the mobile vacancy concentration [23-25]. The increased vacancy concentrations by SPD may lead to pronounced enhancement of diffusion kinetics [24,25]. The excess, non-equilibrium vacancies created by SPD can be understood on the basis of interactions of dislocations, e.g. jog dragging and dipole annihilation [26,27]. As such, the mobile vacancy concentration will be proportional to both the imposed strain rate and the dislocation density $[28,29]$.

Diffusion enhancement by SPD may thus promote the formation of a wide spectrum of highly non-equilibrium states in nc materials, provided other necessary conditions are given. As a result of HPT, for instance, the formation of supersaturated solid solutions in immiscible $\mathrm{Al}-\mathrm{Fe}$ alloys and pearlitic steels [30,31], disordering or even amorphization of intermetallics [32] and alloying in the immiscible $\mathrm{Cu}-\mathrm{Fe}$ composite [24] have been reported. Similar events have been observed in powder mixtures processed by mechanical alloying (MA) [33-36]. In MA of ductile metals, the powder particles trapped between the colliding balls and the vial walls undergo simultaneous deformation, fragmentation and cold welding, resulting in agglomerates of multilayered structures with clean interfaces. Inter-diffusion in such aggregates often leads to an extension of the solid solution limits beyond the equilibrium ones, and the formation of nc alloys, intermetallic compounds and amorphous phases.

A careful examination of the documented investigations related to SPD suggests, however, that one important issue has been largely overlooked, and is therefore poorly understood. This issue pertains to the kinetics of the diffusionrelated processes during SPD. In particular, as we have alluded in the preceding paragraphs, diffusion processes are strongly coupled with generation of crystal defects, such as dislocations, GBs and TJs.

From what we have laid out in the above comes the theme of the present study: SPD-enhanced diffusion and, as a result, formation of nc intermetallic compound and various types of solid solutions. Here we report the production by SMAT of an nc intermetallic layer on the surface of bulk cobalt bases. Our strategy was to incur greatly enhanced diffusion during SPD that leads to a non-equilibrium and rapid alloying process, which eventually leads to the formation of an nc intermetallic compound layer. The microstructural evolution as a function of the imposed strain, including the extension of the solid solutions, phase transformations and formation of intermetallic compounds, was systematically investigated. Discussions are presented by taking into account factors such as grain sizes and various types of crystal defects and their influences on diffusion kinetics.

\section{Experimental procedure}

The base material for SMAT used in this study was an electrodeposited cobalt plate (purity: $99.98 \mathrm{wt} . \%$ ). The average grain size was $\sim 30 \mu \mathrm{m}$ and the dimension of the Co-base was $100 \times 100 \times 5 \mathrm{~mm}^{3}$.

The details of the SMAT processing have been described elsewhere [5,6]. In brief, the Co-base was securely seated in a cylinder-shaped stainless steel chamber that was loaded with a large number of hardened steel balls. The chamber was attached to a vibration generator. During SMAT the steel balls were resonated with the high-frequency vibrations of the system. As a consequence, the energetically moving steel balls impinged the specimen surface continuously, thus imposing strains on the surface layer of the Cobase. This leads to progressive grain refinement. Hence, in much the same way as in MA processing, one would expect enhanced diffusion within the surface layer caused by such deformation processes from the bombarding of the steel balls onto the Co-base surface $[33,36]$. One would also expect the existence of both strain and strain rate gradients as one moves away from the topmost-surface of the SMAT specimen towards the inner depth of the Co-base. Essentially, the topmost surface experiences the largest strain and strain rates which vanish quickly into the depth of the base material. Consequently, a grain size gradient or other microstructural features may develop below the surface of the SMAT sample [5]. This provides a wealth of information regarding the processes that might have taken place within this layer. Therefore, it lends us the opportunity to examine the diffusion processes and their dependence on strains and grain size regimes. In the present work, the SMAT chamber was first evacuated to reduce oxidation of the materials. SMAT was performed for $50 \mathrm{~min}$ in vacuum at room temperature and at a vibrating frequency of $50 \mathrm{~Hz}$. The hardened steel balls had a diameter of $8 \mathrm{~mm}$ and a nominal composition (wt.\%) of $1.35 \mathrm{Cr}$, $0.98 \mathrm{C}$, base $\mathrm{Fe}$.

Following SMAT, the microstructural characterization was performed in a scanning electron microscope (SEM) and a field emission gun transmission electron microscope (FEG-TEM). Both planar-view and cross-sectional thin foils for TEM observations were prepared by means of standard procedures, with special care taken to avoid temperature rise during TEM sample preparation. Careful local compositional analyses were conducted with energy-dispersive X-ray (EDX) spectroscopy by using a very small spot size $(\sim 2 \mathrm{~nm})$ with a typical spectrum collection time of $100 \mathrm{~s}$. X-ray diffraction (XRD) analyses were conducted to identify the phase constitutions within the treated layer. 


\section{Experimental results}

\subsection{Microstructures in the SMAT specimens}

Fig. 1 displays the XRD patterns from the original Cobase and a SMAT specimen. All the peaks from the untreated base can be indexed with the hexagonal closepacked (hcp) lattice of Co. However, these hcp peaks have been almost completely suppressed in the XRD results of the SMAT specimen. The XRD peaks from the SMAT surface can be confidently indexed with a body-centered cubic (bcc) phase, with the lattice constant and $d$-spacing matching those of the intermetallic compound $\mathrm{Co}_{3} \mathrm{Fe}_{7}$. Based on the XRD results, we can conclude that, during the course of SMAT, some alloying processes have occurred on the surface of the Co-base, which eventually result in the formation of the intermetallic compound phase of $\mathrm{Co}_{3} \mathrm{Fe}_{7}$. Also to be noted is the apparent broadening of the XRD peaks of the SMAT specimen, implying very small grain size and/or large microstrains in the specimen.

A cross-section SEM micrograph of the treated layer is shown in Fig. 2. A layer $\sim 20 \mu \mathrm{m}$ thick with contrast distinct from that of the Co-base can be easily observed. This kind of interlayer structure is quite similar to those found in MA [36]. The compositional analyses to be presented support the XRD results given above, namely, the formation of the intermetallic compound of $\mathrm{Co}_{3} \mathrm{Fe}_{7}$. Notice in Fig. 2 the subtle difference in microstructures of the Co-base immediately below the interface and those in the deeper Co-base.

The microstructures below the SMAT surface were investigated by detailed cross-sectional TEM analysis. Fig. 3a and b presents bright- and dark-field TEM images taken from an area presumably $\sim 5 \mu \mathrm{m}$ deep from the top SMAT surface, showing the presence of nc grains. Fig. 3c is the selected-area electron diffraction (SAED) pattern. The set of rings in the SAED pattern can be indexed, as in the case of the XRD peaks given in Fig. 1, with the

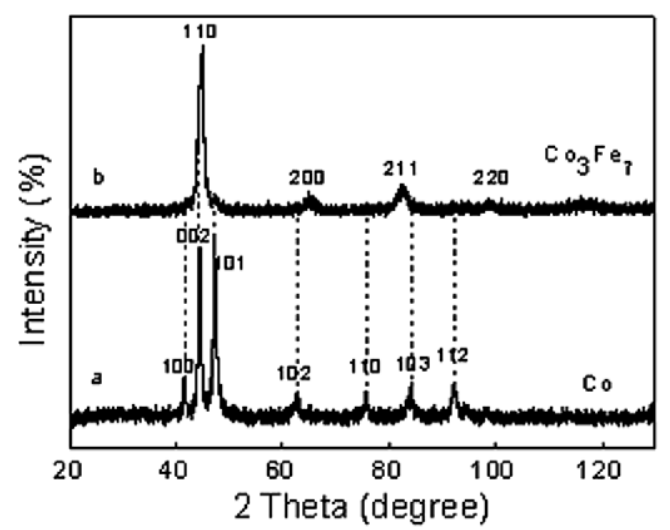

Fig. 1. X-ray diffraction patterns from the un-treated (a: bottom) Co-base and the SMAT specimen (b: upper). Notice that the XRD peaks from the SMAT specimen can be indexed with the bcc phase of the intermetallic compound $\mathrm{Co}_{3} \mathrm{Fe}_{7}$.

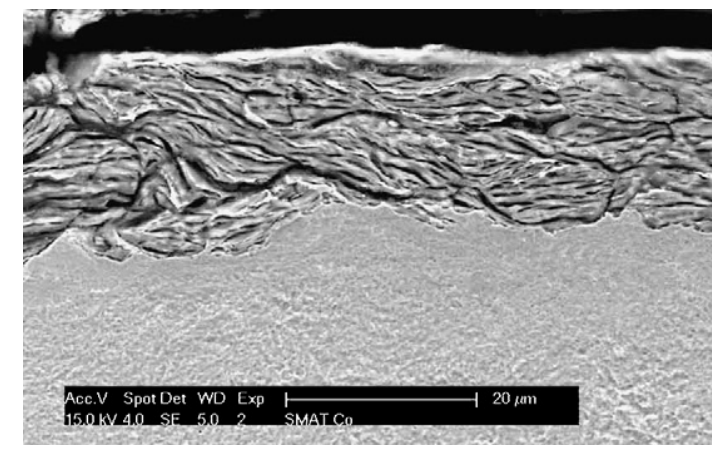

Fig. 2. Transverse microstructure of the treated layer. The roughened, top layer is the top SMAT surface layer with a thickness around $20 \mu \mathrm{m}$. Notice the sharp interface between the roughened top layer and the Co-base.

bcc lattice of the intermetallic compound phase of $\mathrm{Co}_{3} \mathrm{Fe}_{7}$. Fig. 3d shows the high-resolution lattice image of an nc $\mathrm{Co}_{3} \mathrm{Fe}_{7}$ grain which is almost free of lattice defects. The EDX spectrum acquired within this nanometer grain is presented in Fig. 3e, which suggests an Fe-rich phase. Quantitative analyses of the EDX results, as given in the inset of Fig. 3e, again point to the presence of the $\mathrm{Co}_{3} \mathrm{Fe}_{7}$ phase. Also to be noted is the presence of a minor amount of $\mathrm{Cr}$. A very narrow grain size distribution of the intermetallic compound of $\mathrm{Co}_{3} \mathrm{Fe}_{7}$ can be established by the histogram plot of the grain sizes presented in Fig. 3f. The average grain size is found to be $\sim 10 \mathrm{~nm}$, and the majority of the grains have sizes no larger than $10 \mathrm{~nm}$. TEM observations at various depths from the top SMAT surface further confirm the formation of the intermetallic phase of $\mathrm{Co}_{3} \mathrm{Fe}_{7}$ within the treated layer down to a depth of about $\sim 20 \mu \mathrm{m}$ from the top SMAT surface, consistent with the XRD results.

To understand the appearance of the intermetallic phase, the initial stages associated with the formation of this phase was investigated further. Fig. 4a shows the microstructures located in close vicinity of the intermetallic phase/Co-base interface ( $\sim 20 \mu \mathrm{m}$ deep from the top surface). A number of very small $\mathrm{Co}_{3} \mathrm{Fe}_{7}$ grains are observed, together with several large grains. Fig. $4 \mathrm{~b}$ displays the corresponding dark-field TEM micrograph. The inset in Fig. $4 \mathrm{~b}$ is the microdiffraction pattern taken from grain A. The set of diffraction spots along with the rings can be indexed with the bcc $\mathrm{Co}_{3} \mathrm{Fe}_{7}$ phase. It is particularly interesting to examine in more detail grain $\mathrm{A}$ in the darkfield image of Fig. 4b. This apparently single grain actually contains numerous extremely small nc $\mathrm{Co}_{3} \mathrm{Fe}_{7}$ grains, whose sizes are hardly resolvable at such high magnification (note the scale bar of the micrograph). Nanoscale chemical probing of grain A by EDX within the TEM suggests inhomogeneous $\mathrm{Fe}$ distribution, ranging from 16.27 to 57.36 at.\%. This provides strong evidence that the intermetallic $\mathrm{Co}_{3} \mathrm{Fe}_{7}$ phase was nucleated directly from within a bce solid solution. HRTEM observations show that the grain sizes of the $\mathrm{nc} \mathrm{Co}_{3} \mathrm{Fe}_{7}$ grains within grain $\mathrm{A}$ in Fig. 4 are only $\sim 3-5 \mathrm{~nm}$, much smaller than those 

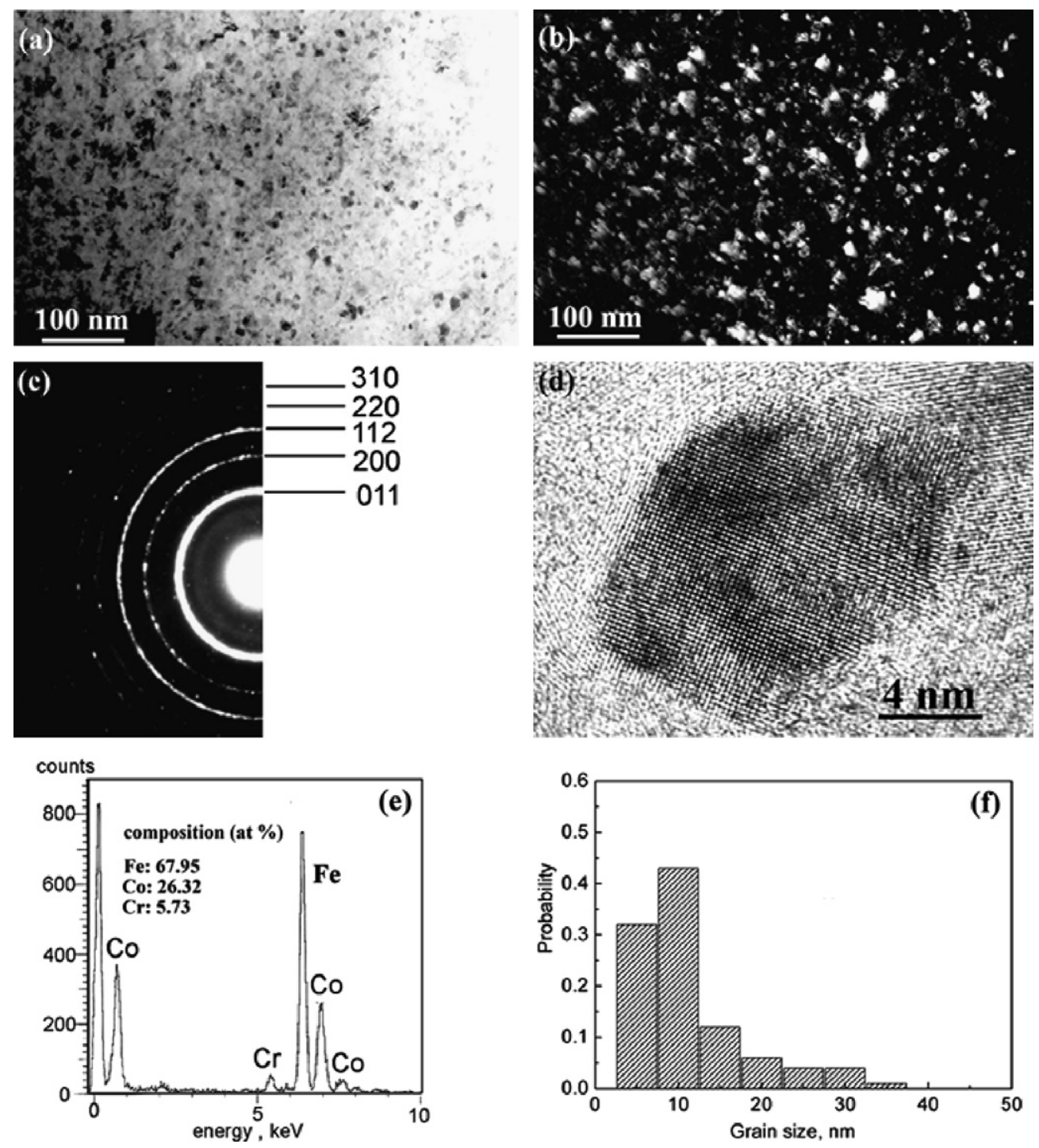

Fig. 3. Bright-field (a) and dark-field (b) cross-sectional TEM micrographs showing the intermetallic phase of $\mathrm{Co}_{3} \mathrm{Fe}_{7}$ at a depth of $\sim 5 \mu \mathrm{m}$ from the topmost SMAT surface. (c) Corresponding SAED pattern. (d) High-resolution lattice image of a $\mathrm{Co}_{3} \mathrm{Fe}_{7}$ grain. The grain is almost perfect. (e) EDX spectrum of a $\mathrm{Co}_{3} \mathrm{Fe}_{7}$ grain, showing the $\mathrm{Fe}$ rich phase composition and the minor existence of $\mathrm{Cr}$. (f) Histogram of grain size distribution of the $\mathrm{Co}_{3} \mathrm{Fe}_{7}$ intermetallic phase.

presented in Fig. 3a, indicative of limited grain growth during SMAT deformation. We therefore believe that both an extension of the solid solution between $\mathrm{Fe}$ and $\mathrm{Co}$ and phase transformations occur prior to the eventual formation of the intermetallic phase, which will be detailed in what follows.

\subsection{Extension of solid solution and phase transformations}

In order to clarify the microstructural evolution pertaining to the formation of the intermetallic compound, the interface between the intermetallic layer and the Co-base was carefully examined via cross-sectional TEM. Fig. 5a is a bright-field TEM micrograph taken from the interface area of the SMAT specimen $(\sim 20 \mu \mathrm{m}$ deep from the top SMAT surface). A clear, drastic change in grain sizes across the interface is observed. The submicron-sized grains on the right-hand side of the micrograph are identified to be the hcp Co. These grains contain many stacking faults (SFs) in their interiors. The nc grains on the lefthand side of the micrograph are identified to be the intermetallic phase of $\mathrm{Co}_{3} \mathrm{Fe}_{7}$. Fig. $5 \mathrm{~b}$ and $\mathrm{c}$ shows the SAED patterns of the bcc $\mathrm{Co}_{3} \mathrm{Fe}_{7}$ and the hcp Co, respectively, both consisting of rings which are continuous for the bcc intermetallic phase and semi-continuous for the hcp Co. Both SAED patterns suggest random orientations of the grains.

Of particular interest is the finding of a "transition zone" with a variety of lattice structures as well as chemical compositions. This transition zone exists between the $\mathrm{Co}_{3} \mathrm{Fe}_{7}$ intermetallic layer and the Co-base. Fig. 6 presents the EDX spectra and the corresponding SAED patterns 

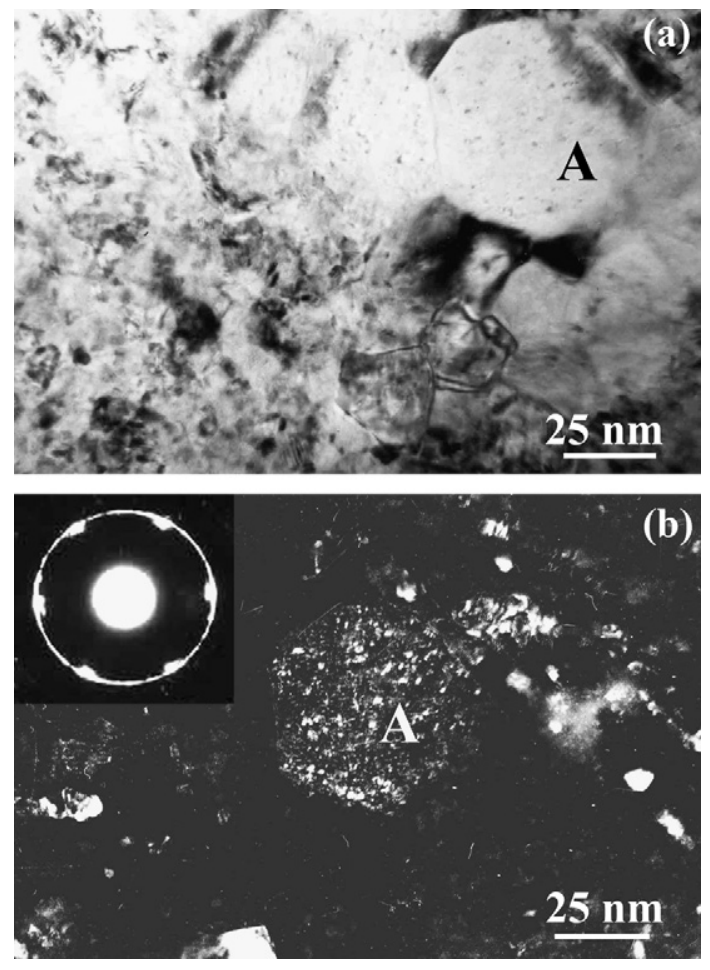

Fig. 4. Bright-field (a) and dark-field (b) cross-sectional TEM micrographs taken at the interface $\sim 20 \mu \mathrm{m}$ deep. Inset in (b) is the microdiffraction from grain A, with the continuous ring superimposed on the spots. Notice particularly the detailed microstructure of grain A in the dark-field image which shows within this nc grain the existence of extremely small nc grains. Presumably grain A is of bcc lattice structure with the spots from the $\langle 111\rangle$ zone (the reflections are of course $\{110\}$ ). Streaks in the $\{110\}$ reflections imply strong lattice strains or the presence of polycrystalline mosaic domains separated by small angle grain boundaries.

obtained in three grains located in three different positions within the SMAT surface layer, with the first (Fig. 6a) closest to the Co-base, the second (Fig. 6b) in between and the last (Fig. 6c) nearest the SMAT top surface. The first diffraction pattern can be easily indexed with the $\langle\overline{1} 2 \overline{1} 6\rangle$ zone of an hcp structure; the second diffraction pattern with the $\langle 110\rangle$ zone of a face-centered cubic (fcc) structure; the third with $\langle 159\rangle$ of a bcc structure. The associated $\mathrm{Fe}(\mathrm{Cr})$ contents (at.\%) as analyzed by EDX within these regions are $1.67(0.23) \%, 9.40(1.42) \%$ and $23.09(1.37) \%$, respectively, indicating a successive extension of solid solution and the occurrence of a series of phase transformations as a result of migration of $\mathrm{Fe}(\mathrm{Cr})$ into the Co-base across the transition zone.

Extensive EDX measurements with high spatial resolution show that cobalt will keep its hcp structure with increased Fe content (at. \%) of up to $\sim 3.9 \%$; it then changes to an fcc structure if the Fe content is $4-11 \%$; finally, it will transform to a bcc structure if the Fe content is further increased. This is in line with the composition and structural analysis results presented in Fig. 6. Accordingly, we may propose the following phase transformation sequence that occurred in the SMAT surface layer as a function of
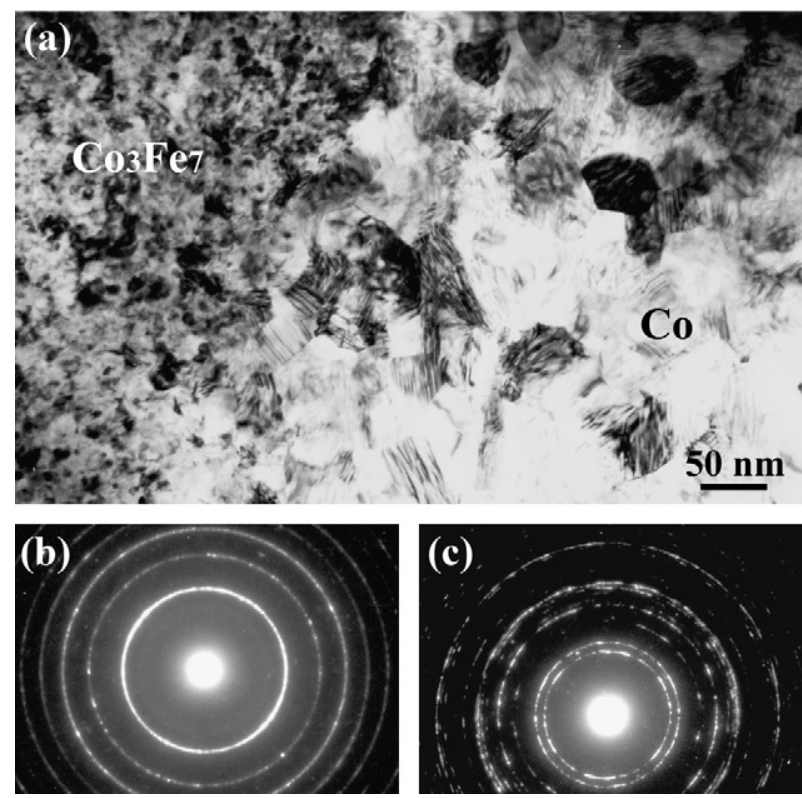

Fig. 5. (a) Cross-sectional TEM micrograph taken in a region near the interface between intermetallic layer and the Co-base $(\sim 25 \mu \mathrm{m}$ deep from the topmost SMAT surface). (b,c) SAED patterns of the intermetallic phase and Co-base. Notice also in (a) the presence of stacking faults in numerous grains of Co which have nanometer sizes.

Fe content (at.\%): hcp $(<4 \% \mathrm{Fe}) \rightarrow$ fcc $(4-11 \% \mathrm{Fe}) \rightarrow$ bcc $(>11 \% \mathrm{Fe})$. Naturally, the degree to which this sequence has actually taken place depends on the SMAT time allowed for the $\mathrm{Fe}$ atoms to migrate into the Co-base. These results show only small deviations from the $\mathrm{Co}-\mathrm{Fe}$ equilibrium phase diagram as it is extrapolated to room temperature regime $[38,39]$. The absence of any intermetallic phase is noteworthy in the Co-Fe binary phase diagram, however. EDX results of Fig. 6 indicate that $\mathrm{Cr}$ contents in the solid solutions with hcp, fcc and bcc structures are minor. They also show that $\mathrm{Cr}$ content is the highest in the bcc phase (nearest the top SMAT surface), and tapers down as one moves deeper into the specimen. We believe the major reason for the minor amount of $\mathrm{Cr}$ in these phases is the much limited source of $\mathrm{Cr}$ available from the hardened steel balls.

\subsection{Chemical compositions at the TJS and GBs, and within the grains}

The EDX composition analyses were made in both the transition zone and intermetallic layer in order to shed light on the nature of diffusion as affected by grain sizes and other defects. Special attention is focused on effects of certain defects on diffusion kinetics. Such defects include SFs, GBs and TJs. Fig. 7a shows deformation-induced high density SFs. These high-density SFs are believed to be the primary defects in the nc and submicron-sized cobalt grains because of the low SF energy of Co [37]. Fig. 7b displays a bright-field TEM micrograph taken right at the interface between the intermetallic compound layer and the Co-base 


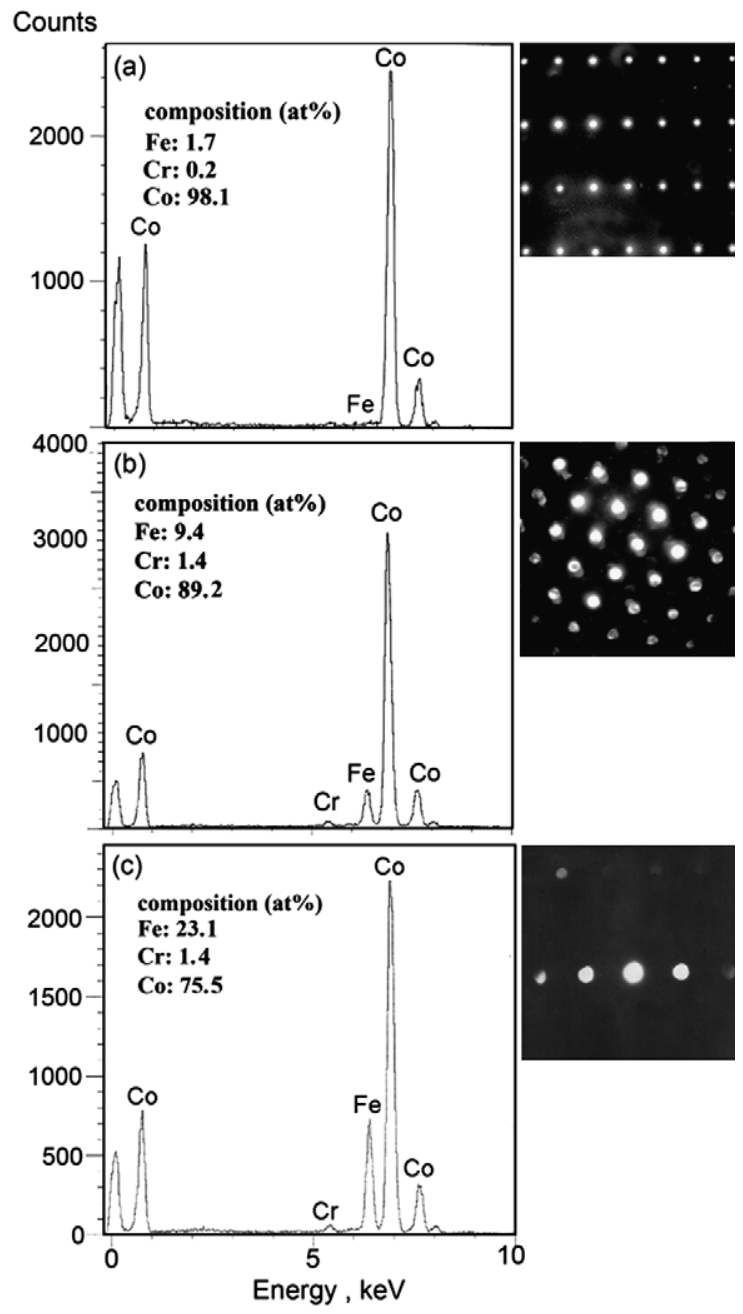

Fig. 6. High-spatial resolution EDX spectra and the corresponding microdiffraction patterns showing the change of the crystal structure from hcp (a) to fcc (b) and to bcc (c) due to an increase in $\mathrm{Fe}(\mathrm{Cr})$ contents within the "transition zone". Also notice that the bcc structure lies closes to the quasi-stoichiometric $\mathrm{Co}_{3} \mathrm{Fe}_{7}$ phase, followed by the fcc structure. The hcp structure is the nearest to the hcp Co-base.

( $\sim 20 \mu \mathrm{m}$ deep from the top SMAT surface). Again SFs are observed in the relatively large hep grains. The presence of a high density of SFs may contribute to enhanced diffusion [40]. Fig. 7c is a TEM micrograph showing the nature of GBs in the SPD specimen. The GBs surrounding a bcc grain (A in Fig. 7c) exhibit diffused contrast, implying the non-equilibrium and high-energy state of this grain produced by severe plastic deformation [30]. It is believed that this kind of GB may strongly enhance the diffusion kinetics of the material $[1,10]$.

Fig. 8 summarizes the overall composition data as evaluated at the TJs, SFs and GBs, and within the grain interiors (GIs) of various phases found in the SMAT layer. A clear trend of the change in $\mathrm{Fe}$ (and also $\mathrm{Cr}$ ) contents at these locations can be established in the following order: GIs $<$ GBs $<$ TJs. Of particular interesting is that, in the hcp phase, higher Fe contents are detected at SFs than at GBs. Fig. 9 shows Fe concentration profiles based on
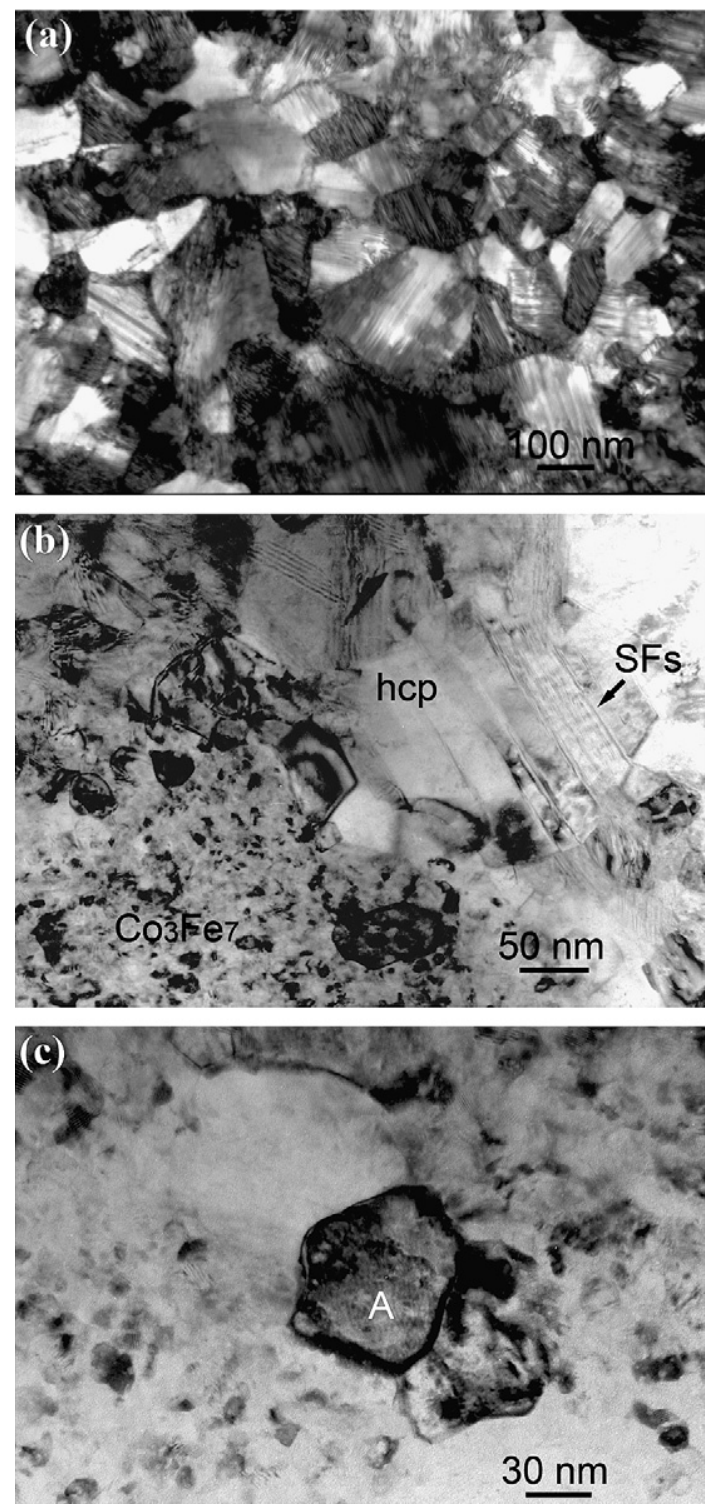

Fig. 7. TEM micrographs showing: (a) high-density SFs of nc and submicron-sized cobalt grains and (b) the interface area between the intermetallic $\mathrm{Co}_{3} \mathrm{Fe}_{7}$ and the Co-base. Notice the presence of SFs in hcp solid solution (based on EDX analysis, not shown here). (c) Nonequilibrium GBs surrounding a bcc grain (labeled by letter A).

more careful, nanoprobe composition analysis near SFs. The significantly high concentrations of $\mathrm{Fe}$ at the SFs strongly suggest the role of SFs as rapid diffusion paths. This implies that solutes segregated to the SFs, or that SFs can serve as fast diffusion paths in much the same way as dislocation cores. In addition, $\mathrm{Fe}$ has been detected both at the GBs and within the grain interiors in the hep grains with grain sizes even as large as $\sim 220 \mathrm{~nm}$. The significance of this observation is twofold. First, diffusion has taken place within the SPD induced ultrafine grains. Second, volume diffusion processes have occurred, the consequence of which may not be neglected as regards their effects on the microstructure and properties of the SMAT specimen. 

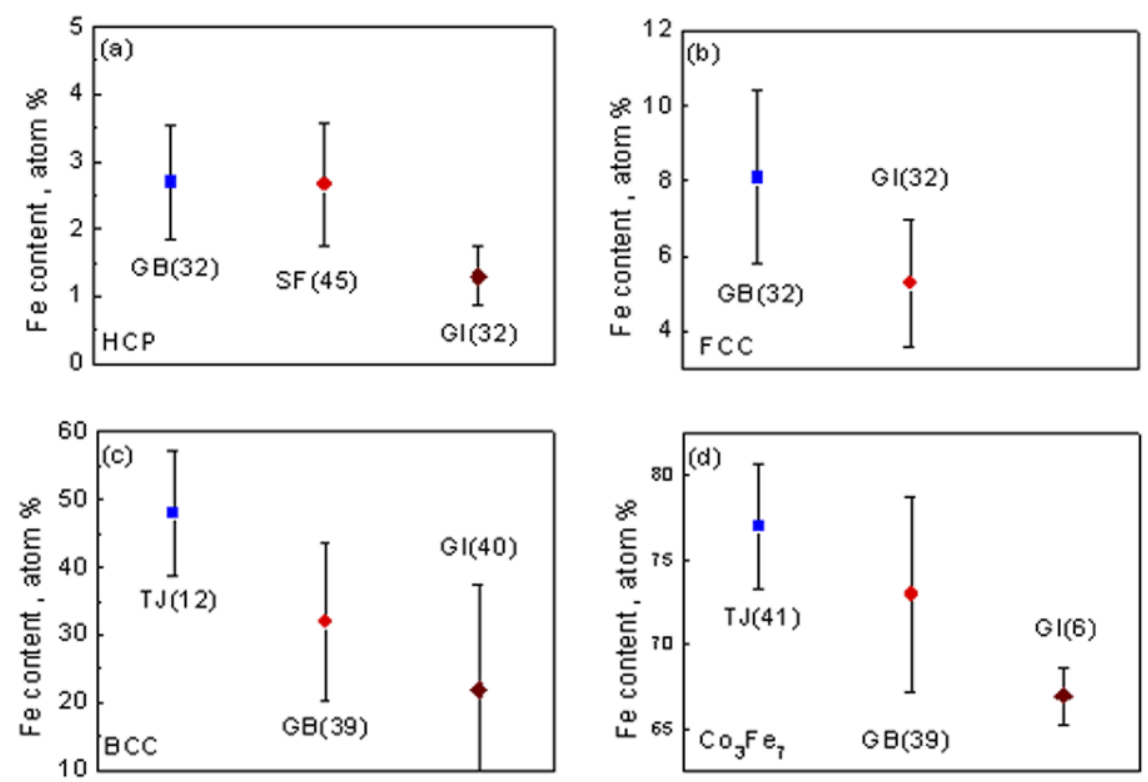

Fig. 8. Nanoprobe EDX compositional analysis results for Fe contents in the stacking faults (SFs), at the grain boundaries (GBs), within the grain interiors (GIs) and at the triple junctions (TJs) in various phases found in the SMAT specimen. Notice that, in the hep phase, Fe contents at the GBs and SFs are higher than those in GIs. It is similar in other phases. Also remarkable is the much higher Fe contents at TJs than at GBs and in GIs. This is true even for the quasi-stoichiometric intermetallic phase of $\mathrm{Co}_{3} \mathrm{Fe}_{7}$.

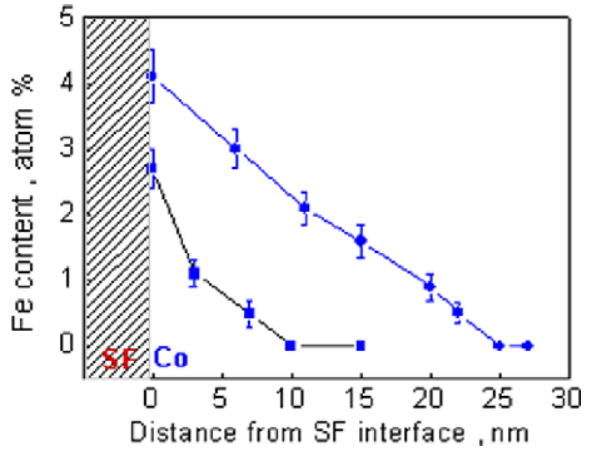

Fig. 9. Distribution of Fe near a SF ribbon from quantitative nanoprobe EDX measurements. The measurements were made along the direction normal to the SF interface. Given in the plot are the mean values and the error bars associated with each location of measurement.

\subsection{Hardness profile of the SMAT layer}

We have used an instrumented nanoindenter to probe the hardness profile over the cross-section of the SMAT specimens. Fig. 10 displays the hardness as a function of the depth from the top SMAT surface. A sharp rise in hardness can be observed at a depth of $\sim 25 \mu \mathrm{m}$ from the top SMAT surface. In other words, a very hard layer with a thickness of $\sim 25 \mu \mathrm{m}$ has been formed on the surface of the SMAT Co. These results are in accordance with microstructural and composition analyses presented in preceding sections in that an intermetallic $\mathrm{Co}_{3} \mathrm{Fe}_{7}$ layer has been found on the SMAT surface of Co. Fig. 10 shows that the hardness of the intermetallic layer ranges from 14 to $12 \mathrm{GPa}$, much higher than the hardness of the cobalt phase near the interface $(\sim 6.2 \mathrm{GPa})$. However, the hardness of

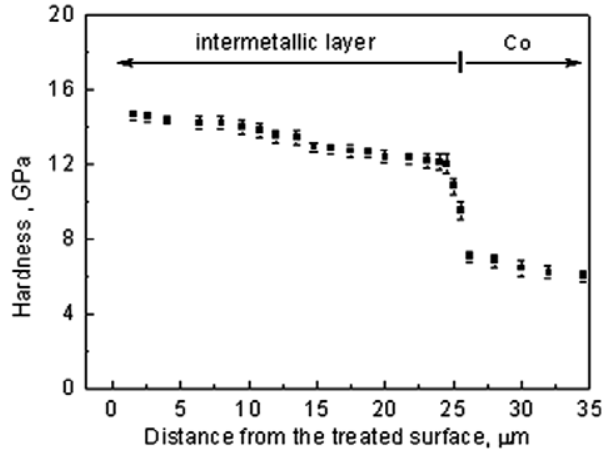

Fig. 10. Hardness profile measured by an instrumented nanoindenter on the cross-section of the SMAT sample. Notice the drastic rise of hardness at a depth of $\sim 25 \mu \mathrm{m}$ from the topmost SMAT surface.

the Co immediately adjacent to the interface is still more than twice that of annealed Co, suggesting a much refined grain size and/or the presence of defects such as dislocations and stacking faults. This is also in agreement with the microstructural analyses of previous sections, where we found that the grain size of Co in the vicinity of the interface is about $\sim 250 \mathrm{~nm}$, and such grains contain high-density SFs.

\section{Discussion}

To begin with, we present in Fig. 11 a schematic to delineate the possible processes occurring during the SMAT processing of the surface of the Co-base. We should point out that such a schematic is based on the experimental results of microstructures and chemical compositions of various regions of the SMAT processed surface. Two 


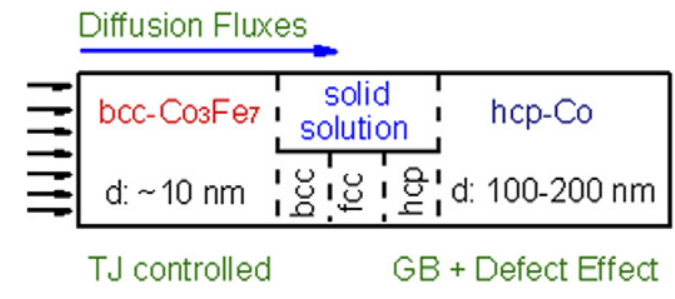

Fig. 11. Schematic illustration of the microstructural evolution as a result of diffusion during the SMAT process. Note the presence of a transition zone containing hcp, fcc and bcc solid solutions, respectively, due to diffusion and phase transformations that have taken place when $\mathrm{Fe}$ contents reached certain levels. See text for detailed discussion.

general processes can be identified with the assistance of this schematic: (1) the extension of solid solution followed by a series of phase transformations (from hcp to fcc, and finally to bcc) in the transition zone; and (2) the formation of the intermetallic compound of $\mathrm{Co}_{3} \mathrm{Fe}_{7}$ at the topmost surface layer of the SMAT specimen. One would naturally believe that these processes involve diffusion of various atomic species during SMAT. Therefore, understanding the atomic-scale diffusion process is the key to the interpretation of the experimental results presented in this paper.

\subsection{Diffusion along GBs, $S F s$ and $T J S$}

The repetitive impingements of the Co-base by the hardened steel balls quickly refine the Co grains and produce fresh ball/specimen surfaces during the SMAT process [37]. Considering the rapid, high-frequency impact from the steel balls, transfer of $\mathrm{Fe}$ and $\mathrm{Cr}$ or other elements to the Co-base from the steel balls and walls of the steel container might take place, as in the case of MA [35,38].

At first, diffusion of $\mathrm{Fe}(\mathrm{Cr})$ should occur from the top surface of the Co-base into the Co grains (with hep lattice structure). As the impinging events of the steel balls on the surface of the Co-base continuously supply $\mathrm{Fe}(\mathrm{Cr})$, this diffusion process continues and the shallow surface layer of the Co-base will be enriched by $\mathrm{Fe}$ in a very short time. This will eventually lead to the formation of the intermetallic compound phase rich in $\mathrm{Fe}$, as confirmed by the XRD, TEM and compositional analyses presented in the preceding sections. As the SMAT process continues, the intermetallic compound layer will extend into the depth of the Co-base. Quantitative EDX (Fig. 3e) suggests that the composition of the intermetallic phase is quite close to that of the $\mathrm{Co}_{3} \mathrm{Fe}_{7}$ compound, which indeed has a bcc structure. Away from the quasi-stoichiometric $\mathrm{Co}_{3} \mathrm{Fe}_{7}$ phase toward the interior of the Co-base, the contents of Fe should taper down, and vanish at a certain depth. As such, a concentration profile is established. Depending on the Fe concentrations, different lattice structures are formed. Based on the experimental results, we presume the following phases in the "transition zone": hcp (Fe content $<4$ at.\%) immediately above the interface; fcc (Fe content between $\sim 4$ and 11 at.\%) in between; and bcc (Fe content >11 at.\%) between the fcc phase and the quasi-stoichiometric $\mathrm{Co}_{3} \mathrm{Fe}_{7}$ intermetallic phase. The existence of these phases in the transition zone has been established by high-spatial-resolution EDX probing as well as microdiffraction analysis. The hcp phase within the transition zone has ultrafine grain sizes, whereas the fcc and bcc phases have grain sizes within the range of $50-100 \mathrm{~nm}$, namely, they are nanocrystalline phases. Composition data in Fig. 8 show that the Fe contents in the GBs are much higher than those in the grain interiors. As was pointed out formerly, the GBs of the SPD-induced fine grains are characterized by a highly non-equilibrium and high-energy state (see e.g. Fig. 7c). This high-energy state associated with such GBs qualifies them as diffusion "short-circuits" such that defect formation energy may be lowered $[13,14]$. Furthermore, it has been recognized that GB diffusion in SPD-produced materials is determined primarily by the GB state rather than by the mean grain size [7]. Hence, fast GB diffusion may occur even when grain sizes are above the nanometer scale, so long as the GBs maintain their high-energy, non-equilibrium nature. Meanwhile, due to the much higher volume fraction of GBs than that of TJs for relatively large grains, it is reasonable to believe that GB diffusion should dominates in the transition zone [17].

Another point of particular interest is associated with the high-density SFs found in the hep Co grains in the proximity of the interface, as displayed by Figs. 5 and 7. Nanoprobing results presented in Figs. 8 and 9 show that $\mathrm{Fe}$ contents in SFs are comparable to those at the GBs, and both higher than those in the GIs. This indicates that SFs may contribute to fast diffusion, too, as pointed out by Wuttig and Birnbaum [40]. Our experimental results attest to the molecular dynamics (MD) simulations by Huang and co-workers who found pipe diffusion in the partials' cores of SFs due to decrease in the formation energy of defects near the SFs [41]. In the present case, diffusion is also closely coupled with continual formation of SFs due to the SMAT processing. Therefore, diffusion is further enhanced by stresses due to the repetitive bombarding of the hardened steel balls. We hence believe that diffusion along the GBs and SFs are the primary mass transfer modes occurring in the hep Co grains.

The formation of a continuous layer of $\mathrm{Co}_{3} \mathrm{Fe}_{7}$ intermetallic phase might first make one think that the diffusion will slow down, as is the case of oxidation of $\mathrm{Si}$, where a dense, continuous layer of amorphous $\mathrm{SiO}_{2}$ phase lowers the oxidation rate by reducing the oxygen transport rate into the $\mathrm{Si}$ matrix. However, since the grain size of the intermetallic phase is only $\sim 10 \mathrm{~nm}$, the volume fraction of both GBs and TJs will be very significant [20]. Recent experiments by Bokstein and co-workers have demonstrated that $\mathrm{Zn}$ diffusivity along TJs in $\mathrm{Al}$ is three orders of magnitude higher than that along the GBs [18]. Diffusion mechanism maps constructed by Chen and Schuh also showed that TJ diffusion should be the dominant mechanism within this grain size regime [17]. According to Chen and Schuh [17], the volume fraction of GBs and TJs in nc materials can be written as 
$f_{\mathrm{GB}}=H_{\mathrm{GB}} \frac{\delta}{d}(\mathrm{a})$ and $f_{\mathrm{TJ}}=H_{\mathrm{TJ}}\left(\frac{\delta}{d}\right)^{2}$

where $\delta$ is the thickness of GBs and $d$ is the grain size; $H_{\mathrm{GB}}$ and $H_{\mathrm{TJ}}$ are dimensionless geometric parameters depending on the grain shape and grain size distribution. For Voronoi polyhedra, which have nearly log-normal grain size distribution, $H_{\mathrm{GB}}$ and $H_{\mathrm{TJ}}$ are 2.9105 and 2.5259, respectively. An effective GB thickness can be assumed to be $\sim 1.0 \mathrm{~nm}$.

Therefore, within the thin intermetallic compound layer of $\mathrm{Co}_{3} \mathrm{Fe}_{7}$ with grain size $\sim 10 \mathrm{~nm}$, the volume fractions of GBs and TJs are $\sim 30 \%$ and $\sim 3 \%$, respectively. This evaluation is close to that using the equations given in Ref. [20].

It has been recognized that intercrystalline diffusion dominates in polycrystalline metals at low homologous temperatures [42]. The conventional way of including contributions from GB and TJ diffusion is by extending the Hart equation [43] for the apparent (or effective) diffusion coefficient [19]. This approach is essentially based on the rule-of-mixture, which only represents the limiting parallel geometry and ignores the important possibility of lateral interconnections between the fast diffusion paths. Therefore, it must be considered as an upper bound on the apparent diffusivity [17]. Based on recent experimental results on the contribution of TJs to diffusion in polycrystalline materials [18], Chen and Schuh revisited this important problem by geometric considerations, and constructed a more practical model $[17,44]$. Here, the intercrystalline regions consist of GBs and TJs. The GB facets are separated from each other by their adjoining TJs, which form an interconnected, percolating network throughout the specimen [17]. Chen and Schuch derived the following equation for the intergranular diffusivity $D_{\mathrm{IG}}$ :

$D_{\mathrm{IG}}=D_{\mathrm{GB}}+\frac{D_{\mathrm{TJ}}^{2}-D_{\mathrm{GB}}^{2}}{D_{\mathrm{GB}}+\left(1+2 f_{\mathrm{GB}} / f_{\mathrm{TJ}}\right) D_{\mathrm{TJ}}}$.

In Eq. (2), $D_{\mathrm{TJ}}$ and $D_{\mathrm{GB}}$ are the diffusivities along the TJs and GBs, respectively. According to the preceding calculations of the volume fractions of GBs and TJs based on Eq. (1) for $10 \mathrm{~nm}$ grain size, and considering that at a given temperature, $D_{\mathrm{TJ}} / D_{\mathrm{GB}}$ is $\sim 10^{3}, \quad D_{\mathrm{IG}} \approx\left(D_{\mathrm{GB}}+D_{\mathrm{TJ}} / 21\right)$, which is still dominated by the TJ term, in keeping with the analyses by Chen and Schuh in their recent work [44]. We therefore believe that in the $\mathrm{nc} \mathrm{Co}_{3} \mathrm{Fe}_{7}$ intermetallic layer (grain size $\sim 10 \mathrm{~nm}$ ) TJ diffusion provides the predominant fluxes of atoms through this layer.

\subsection{Superimposed effect of deformation on diffusion}

SMAT process induces high-rate deformation in the surface layer of the material being treated. High strain rates are expected to expedite diffusion of migrating species $[24,28,29]$. Diffusion may also be enhanced by deformation-induced "mechanical interdiffusion" [45]. As such, there may exist two driving mechanisms, namely, concentration-gradient-based and strain-potential-gradient-based.
The strain potential, $\mu_{\mathrm{str}}$, defined as a function of strain, $\varepsilon(z)$, at an arbitrary depth $(z)$ of the treated layer, varies with $z$. This variation (or gradient) also contributes to driving the diffusion events.

Hence, the total effective diffusion flux inward the Cobase, $J_{\text {total }}$, can be obtained by including concentration gradient and the strain potential gradient,

$$
\begin{aligned}
J_{\text {total }} & =f_{\mathrm{IG}} J_{\mathrm{IG}}+\left(1-f_{\mathrm{IG}}\right) J_{\mathrm{V}}+J_{\text {str }} \\
& =-D_{\mathrm{IG}} \frac{\mathrm{d} C}{\mathrm{~d} z}-D_{\mathrm{V}} \frac{\mathrm{d} C}{\mathrm{~d} z}-D_{\mathrm{str}} \frac{\mathrm{d} \mu_{\mathrm{str}}}{\mathrm{d} z}
\end{aligned}
$$

where $J_{\mathrm{IG}}, J_{\mathrm{V}}$ and $J_{\mathrm{str}}$ are fluxes due to the intergranular regions (TJs and GBs), volume diffusion solely from concentration gradient and the strain potential gradient, respectively; $C$ is the concentration of the migrating species at a certain depth; $D_{\mathrm{V}}$ is the volume (lattice) diffusion coefficient; and $D_{\text {str }}$ is the diffusion coefficient due to the strain potential gradient. The strain potential gradient may be considered in combination with the effect of stresses on diffusion. It has been shown that the mobility of diffusion species increases linearly with the stresses [46].

In what follows, we will discuss the effect of strain potential gradient on the overall effective diffusion kinetics under SMAT. Three aspects of the phenomenon will be addressed. The first is associated with the dislocations generated during the high-rate deformation of the SMAT surface layer as a result of continual impingements of the high-velocity hardened steel balls. The second is to account for the excess vacancies created during the high-rate severe plastic deformation within the surface layer by SMAT. Finally, local adiabatic heating from the high-rate plastic deformation and its effect on the diffusion kinetics will be discussed.

\subsubsection{Dislocation solute-pumping}

In the early stage of the treating process, SMAT rapidly incurs local but severe plastic deformation in the treated surface of the Co-base $[5,37]$. While new dislocations and other defects are generated, the dislocations within the base will be continually forced to glide, akin to the case in MA [47]. At the same time, diffusion of the various species from the impacting media into the Co-base also begins. The coupling of diffusion and dislocation activity at high strain rates gives rise to a complex picture of the whole process involved. Due to the more open structure and high-energy state of the dislocation cores, dislocations have been considered to be both hosts for impurities and solute atoms, and solute-pumping channels. Dislocations moving under external stresses drag the impurity and solute atoms existing in the core region. At relatively high temperatures, this dragging effect can serve as a non-diffusive, collective transport mechanism [48]. Eckert et al. [49] and Schwarz [50] have proposed the mechanisms for mechanical alloying on the basis of interactions between the dislocation strain fields and the migrating solutes. Energy surplus from the solute-core interaction and the relatively open structure 
of the cores cause the solutes to diffuse along the cores, thus populate the cores by forming linear solute chains. Upon a stress pulse associated with the ball impact, dislocations within the Co-base may be displaced, leaving behind a string of solutes in an otherwise perfect lattice, and are hence available as "diffusion pumps" again. Locally, the lattice is left in a state of high super-saturation. Diffusion in this core region is fast enough for solutes ( $\mathrm{Fe}, \mathrm{Cr}$, etc.) to enter in large quantities. This dislocation solute-pumping mechanism contributes in part to the formation of extended solid solutions. This mechanism may operate over a space up to hundreds of nanometers in size [51]. Since its efficiency is proportional to the dislocation density for pumping effects, and to the velocity and density of mobile dislocations for the dragging effects, the resulting effect should be a function of the strain rate [52]. In the present case, within the relatively large grains in the transition zone (Fig. 11), especially in ultrafine-grained (UFG) hcp Cobase with evident dislocation activities as shown in Fig. 7a, we envision that this mechanism may contribute to the enhanced diffusion.

\subsubsection{Mobile vacancy concentration}

Plastic deformation has been found to substantially increase mobile vacancy concentrations $[24,25]$ in a metallic material. The vacancy formation energy and internal stress are the two primary factors controlling vacancy formation. The former is significantly lowered in nc grains [53]. The internal stresses due to HPT are believed to produce excess vacancies by reducing the vacancy formation energy [54]. The rate of vacancy production is also found to be proportional to the imposed strain rate. Kiritani et al. [55] showed that at room temperature and at a strain rate of $\sim 1.0 \mathrm{~s}^{-1}$ vacancy concentration in copper increases to $10^{-5}$ within $1.0 \mathrm{~s}$, leading to the formation of vacancy clusters. During the HPT process of a $\mathrm{Cu}-\mathrm{Fe}$ filamentary composite, the mobile vacancy concentration and vacancy diffusion coefficient are estimated to increase by 15 and four orders of magnitude, respectively, providing an additional mobile vacancy production rate of $10^{-5} \mathrm{~s}^{-1}$ [24]. The presence of high concentrations of vacancies will naturally increase the overall diffusion coefficient. This has been held responsible for the atomic level alloying observed in the HPT processed composites. The strain rate during SMAT could reach $10^{3}-10^{4} \mathrm{~s}^{-1}$ [5], much higher than accessible via HPT. It is therefore reasonable to believe that the mobile vacancy concentration is greatly increased in the SMAT surface layer. This, in turn, enhances the diffusion kinetics of the various species.

\subsubsection{Temperature rise during deformation}

Adiabatic temperature rise, local or global, is generally anticipated for high-rate plastic deformation [56]. Under dynamic mechanical loading, the heat generated by the mechanical work may not have sufficient time to be dissipated, and the material will experience local or global adiabatic heating. Such a scenario should apply to the
SMAT process, as well as to MA $[33,36]$. The temperature rise will undoubtedly have a strong influence on the diffusivity of solutes and the generation of defects. However, it appears difficult to evaluate the exact temperature change on the top SMAT surface due to a lack of precise information regarding the strains and stresses in that region. In spite of the subtle differences between SMAT and MA, one may still estimate the temperature rise associated with SMAT by using the thermal analyses for MA. Experimental results showed that the overall temperature rise during MA can be as high as $\sim 215^{\circ} \mathrm{C}$. However, local temperatures may rise much higher [36]. Based on the microstructure and chemical analyses presented in this article, the adiabatic temperature rise during the SMAT of Co must not be very high, because otherwise grain growth, recovery of the defected structures, etc. might have occurred. Nonetheless, since diffusion kinetics is an exponential function of inverse temperature (i.e. an Arrhenius-type relation), even a moderate temperature rise will greatly enhance diffusion.

\subsection{Formation of the intermetallic phase}

In the MA of ductile alloy systems with a negative heat of mixing, the mechanism of the formation of metastable intermediate phases has been explained by interdiffusion reactions of the various components [33]. The Co-Fe system has a relatively small negative heat of mixing $\left(\sim-1.0 \mathrm{~kJ} \mathrm{~mol}^{-1}[57]\right)$. Previous work on the MA of elemental $\mathrm{Fe}$ and Co powders reported the formation of the stoichiometric intermetallic phase of $\mathrm{Fe}_{50} \mathrm{Co}_{50}$ [58] as a result of the mixing of the elements by interdiffusion [59]. We propose that, based on the experimental results of the present study, true alloying on the atomic level can be achieved through two mechanisms operating concurrently and complementarily, namely, gradual diffusive intermixing and discontinuous mechanically driven intermixing. We believe that the deformation-assisted diffusion processes play a significant role in controlling the alloying process. The formation of the nc intermetallic $\mathrm{Co}_{3} \mathrm{Fe}_{7}$ is a direct consequence of numerous nucleation events with limited grain growth, as demonstrated by Fig. 4. It is also conceivable that the continuous intermixing of constituents will extend the intermetallic layer, and move the transition zone towards the deeper Co-base.

This work has shown a new route, as schematically delineated in Fig. 12, that can be used for non-equilibrium and fast surface modifications by alloying on the surface of metallic materials. This route eliminates the complex, multiple-stage preparation of intermetallic materials. It allows the synthesis of novel nc materials, dependent on the judicious selection of relevant parameters. Such relevant and important parameters include the composition of the impinging media (the chemistry of the balls), the base materials to be modified, and the environment and vacuum condition of the SMAT chamber. One can regard this technique as an alternate and complementary method of 


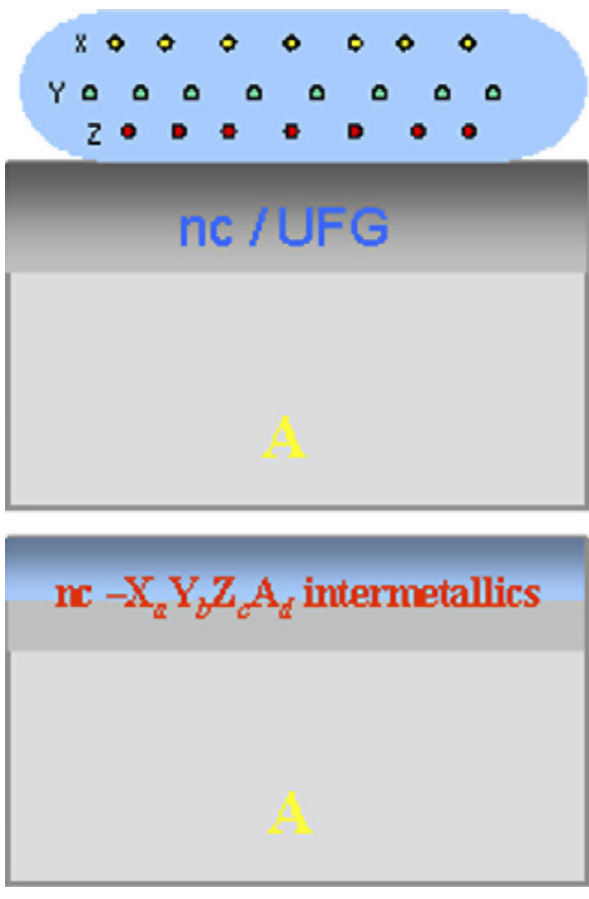

Fig. 12. Schematic illustration to delineate the new surface nanocrystallization route through surface alloying. The top image shows the accumulation of elements (X, Y and Z) from the bombarding media on the surface of the SMAT base (A). The bottom image displays the final condition of the treated specimen with a top layer consisting of an intermetallic phase $\left(\mathrm{X}_{\mathrm{a}} \mathrm{Y}_{\mathrm{b}} \mathrm{Z}_{\mathrm{c}} \mathrm{A}_{\mathrm{d}}\right)$ of super-high hardness, and perhaps various solid solutions with different structures depending on the specific solutes and their concentrations present in the layers.

surface nanocrystallization, in addition to the three processes listed in Ref. [5].

\section{Conclusions}

We have reported systematic experimental investigations on the diffusion and alloying process during SMAT of a Co-base. The major findings and conclusions are summarized below.

Nanocrystalline intermetallic compound $\mathrm{Co}_{3} \mathrm{Fe}_{7}$ (grain size $\sim 10 \mathrm{~nm}$ ) was generated in the treated layer by SMAT of bulk Co-base. This intermetallic compound layer has a bcc structure, as confirmed by XRD and TEM analyses. Through detailed TEM (and especially microdiffraction) and compositional analyses (using a nanoprobe), a "transition zone" was found between the $\mathrm{Co}_{3} \mathrm{Fe}_{7}$ intermetallic layer and the hcp Co-base where the extension of solid solution and a series of phase transformations have occurred. We envision, based on the experimental results, that the sequence of phase transformations depends on $\mathrm{Fe}$ content (at.\%) and follows the order: from hep $(<4 \%$ $\mathrm{Fe})$ to fcc $(4-11 \% \mathrm{Fe})$ and finally to bcc $(>11 \% \mathrm{Fe})$. In this sequence, the bcc phase was observed closest to the top SMAT surface and the hcp phase nearest to the Co-base, with the fcc phase in between.

The hardness profile of the cross-section of the SMAT specimen shows a precipitous rise of hardness to $\sim 13$
GPa beyond the interface between the intermetallic layer and the Co-base. The Co immediately below the interface has ultrafine grains, with a hardness value more than twice that of the conventional grain size. A large density of stacking faults was also observed within those UFG Co grains.

Diffusion of $\mathrm{Fe}, \mathrm{Cr}$ and other species from the hardened steel balls into the Co-base has taken place to form various solid solutions, with the topmost layer being the intermetallic compound $\mathrm{Co}_{3} \mathrm{Fe}_{7}$. Nanoscale composition analyses by EDX showed that $\mathrm{Fe}$ contents are significantly higher in the GBs and TJs than in the GIs. We therefore believe that fast diffusion along GBs and TJs dominate in the nc intermetallic compound. We also believe that the high-density SFs could contribute to the accelerated diffusion during the SMAT process. The combined effects of high-rate deformation on diffusion kinetics were analyzed in terms of a dislocation solute-pumping mechanism and much increased concentration of mobile vacancies, along with adiabatic temperature rise as a result of the high-rate plastic deformation.

Based on the experimental results and the discussions, we envisage that the alloying process was ascribed to intermixing at the atomic scale during high-rate severe plastic deformation. The formation of the intermetallic phase was a consequence of numerous nucleation events followed by limited growth.

\section{Acknowledgements}

This research project was supported by the National Natural Science Foundation of China under Grant Nos. 50471086 and 50571110, National Key Basic Research and Development Program of China under Grant No. 2004CB619305, the Chinese Academy of Sciences under Grant No. KJCX2-YW-M04 and Innovation Program.

\section{References}

[1] Valiev RZ, Islamgaliev RK, Alexandrov IV. Prog Mater Sci 2000;45:103

[2] Segal VM. Mater Sci Eng A 1999;A271:322.

[3] Saito Y, Utsunomiya H, Tsuji N, Sakai T. Acta Mater 1999;47:579.

[4] Hughes DA, Hansen N. Phys Rev Lett 2001;87:135503-1.

[5] Lu K, Lu J. J Mater Sci Technol 1999;15:193; Lu K, Lu J. Mater Sci Eng A 2004;375-77:38.

[6] Tao NR, Sui M, Lu J, Lu K. NanoStruct Mater 1999;11:433.

[7] Wurschum R, Brossmann U, Schaefer H-E. In: Koch CC, editor. Nanostructured materials. Norwich (NY): Noyes Pubs; 2001. p. 267.

[8] Baro MD, Kolobov Yu R, Ovid'ko IA, Schaefer HE, Straumal BB, Valiev RZ, et al. Rev Adv Mater Sci 2001;2:1.

[9] Wurschum R, Herth S, Brossmann U. Adv Eng Mater 2003;5:365.

[10] Kolobov YR, Grabovetskaya GP, Ivanov MB, Zhilyaev AP, Valiev RZ. Scripta Mater 2001;44:873.

[11] Brossmann U, Wurschum R, Sodervall U, Schaefer H-E. J Appl Phys 1999;85:7646.

[12] Tanimoto H, Pasquini L, Prummer R, Kronmuller H, Schaefer H-E. Sci Mater 2000;42:961.

[13] Schaefer H-E, Reimann K, Straub W, Phillipp F, Tanimoto H, Brossmann U, et al. Mater Sci Eng A 2000;286:24.

[14] Tong WP, Tao NR, Wang ZB, Lu J, Lu K. Science 2003;299:686. 
[15] Wang ZB, Tao NR, Tang WP, Lu J, Lu K. Acta Mater 2003;51:4319.

[16] Wang ZB, Lu J, Lu K. Acta Mater 2005;53:2081.

[17] Chen Y, Schuh CA. J Appl Phys 2007;101:063524.

[18] Bokstein B, Ivanov V, Oreshina O, Peteline A, Peteline S. Mater Sci Eng A 2001;302:151.

[19] Wang HT, Yang W, Ngan AHW. Scripta Mater 2005;52:69.

[20] Palumbo G, Thorpe SJ, Aust KT. Scripta Metall Mater 1990;24:1347.

[21] Turnbull D, Hoffman RE. Acta Mater 1954;2:419.

[22] Ballufi RW, Granato AV. In: Nabarro FRN, editor. Dislocations in solids. North Holland; 1979. p. 1-133.

[23] Van Petergem S, Dalla Torre F, Segers D, Van Swygenhoven H. Scripta Mater 2003;48:17.

[24] Sauvage X, Wetscher F, Pareige P. Acta Mater 2005;53:2127.

[25] Straumal BB, Baretzky B, Mazilkin AA, Phillipp F, Kogtenkova OA, Volkov MN, et al. Acta Mater 2004;52:4469.

[26] Ruoff AL, Balluffi RW. J Appl Phys 1963;34:2862.

[27] Friedel J. Dislocations. Oxford: Pergamon Press; 1964.

[28] Murty KL, Detemple K, Kanert O, Dehosson JTM. Metal Mater Trans A 1998;29:153.

[29] Militzer M, Sun WP, Jonas JJ. Acta Metal Mater 1994;42:133.

[30] Nazarov AA, Romanov AE, Valiev RZ. Acta Metall Mater 1993;41:1033.

[31] Ivanisenko Yu, Lojkowski W, Valiev RZ, Fecht H-J. Acta Mater 2003;51:5555.

[32] Korznikov AV, Dimitrov O, Korznikova GF, Dallas J-P, Quivy A, Valiev RZ. NanoStruct Mater 1999;11:17.

[33] Koch CC. NanoStruct Mater 1993;2:109; Koch CC. NanoStruct Mater 1997;9:13;

Koch CC, Whittenberger JD. Intermetallics 1996;4:339.

[34] Fecht H-J. NanoStruct Mater 1995;6:33.

[35] Ma E. Prog Mater Sci 2005;50:413.

[36] Suryanarayana C. Int Mater Rev 1995;40:41; Suryanarayana C. Prog Mater Sci 2001;46:1.
[37] Wu X, Tao N, Hong Y, Liu G, Xu B, Lu J, et al. Acta Mater 2005;53:681.

[38] Mattoso N, Fernandes V, Abbate M, Schreiner WH, Mosca DH. Electrochem Solid-State Lett 2001;4:C20.

[39] Massalski TB, editor. Binary alloy phase diagrams, vol. 2. Materials Park $(\mathrm{OH})$ : ASM International; 1996.

[40] Wuttig M, Birnbaum KH. Phys Rev 1966;147:495.

[41] Huang J, Meyer M, Pontikis V. Phys Rev Lett 1989;63:628.

[42] Shewmon PG. Diffusion in solids. New York: McGraw-Hill; 1963.

[43] Hart EW. Acta Metall 1957;5:597.

[44] Chen Y, Schuh CA. Scripta Mater 2007;57:253.

[45] Balluffi RW, Rouff AL. Appl Phys Lett 1962;1:59.

[46] Larche FC, Voorhees PW. Defect Diffus Forum 1996;129-130:31.

[47] Schwarz RB. Phys Rev B 1980;21:5617.

[48] Hirth JP, Lothe J. Theory of dislocations. Malabar (FL): Krieger Publishing Company; 1992.

[49] Eckert J, Holzer JC, Krill III CE, Johnson WL. J Appl Phys 1993;73:131. and 2794.

[50] Schwarz RB. Mater Sci Forum 1998;269-272:665.

[51] Estrin Y, Rabkin E. Scripta Mater 1998;39:1731.

[52] Embury JD, Deschamps A, Brechet Y. Scripta Mater 2003;49:927.

[53] Qi WH, Wang MP. J Mater Sci 2004;39:2529.

[54] Sato K, Yoshiie Y, Satoh Y, Kuramoto E, Kiritani M. Radiat Effects Defects Solids 2002;157:171.

[55] Kiritani M, Yasunaga K, Matsukawa Y, Komatsu M. Radiat Effects Defects Solids 2002;157:3.

[56] Meyers MA. Dynamic behavior of materials. Wiley Interscience; 1994.

[57] Niessen AK, de Boer FR, Boom R, de Chatel PF, Mattern WCM, Miedema AR. CALPHAD 1983;7:51.

[58] Elkalkouli R, Grosbras M, Dinhut JF. NanoStruct Mater 1995;5:733.

[59] Bruning R, Samwer K, Kuhrt C, Schultz L. J Appl Phys 1992;72:2978. 